\title{
Models of the energy landscape for an element of shakti spin ice
}

\author{
U. B. Arnalds ${ }^{1}$, S. Y. Liashko ${ }^{1,2}$, P. F. Bessarab ${ }^{1,2}$, V. M. Uzdin ${ }^{2,3}$, H. Jónsson ${ }^{1,4,5}$ \\ ${ }^{1}$ Science Institute, University of Iceland, 107 Reykjavík, Iceland \\ ${ }^{2}$ ITMO University, Kronverkskiy, 49, St. Petersburg, 197101, Russia \\ ${ }^{3}$ St. Petersburg State University, St. Petersburg, 198504, Russia \\ ${ }^{4}$ Faculty of Physical Sciences, University of Iceland, 107 Reykjavík, Iceland \\ ${ }^{5}$ Dept. of Applied Physics, Aalto University, Espoo, FI-00076, Finland \\ v_uzdin@mail.ru
}

\begin{abstract}
Micromagnetic calculations are compared with faster model calculations of interacting nanoscopic magnetic islands representing an element of a shakti spin ice lattice. Several pathways for transitions between equivalent ground states are studied. The model calculations describe the interaction between the islands either with the point dipole approximation, or with a dumbbell approximation where the distance between the two poles is optimized to match the micromagnetic results. The closest agreement in the energy of both local minima as well as transition state configurations where one macrospin has rotated by $90^{\circ}$ is obtained with a dumbbell model where the distance between the poles is ca. $20 \%$ smaller than the island length.
\end{abstract}

Keywords: magnetic islands, activation energy, micromagnetics, dipole interaction, dumbbell, spin ice.

Received: 11 November 2018

Revised: 18 November 2018

\section{Introduction}

Artificial spin systems have made it possible to study magnetic ordering phenomena and thermal dynamics at macroscopic length scales in extended spin arrays. These systems consist of lithographically patterned magnetic islands arranged in various ways. Initially, the arrangements were chosen to mimic pyrochlore spin ice [1] but the vast capabilities available within nanostructuring have allowed new research into a multitude of different types of systems [2-5]. The interaction between the islands affects the properties of the artificial spin ice systems controlling their ground state magnetic ordering and affecting the energy barriers for transitions between various possible orientations of the magnetization of the islands. The arrangement of the islands, therefore, directly affects the energetics of the system.

The shakti lattice is a recent addition to the catalog of artificial spin ice systems. Extended systems of this geometry have been studied both theoretically [6,7] as well as experimentally $[9,10]$. Interestingly, the shakti geometry falls into a category of pentagonal tilings but is often described as a decimation of a square tiling. A closer analogy would be that of a Cairo pentagonal tiling or a Panama weave tiling as the system is composed of islands of two different lengths [6]. The different island lengths give rise to different energy barriers for reversing their magnetization direction creating an energy scale hierarchy affecting the energy landscape and ordering of the system [10].

Studies of finite arrays of islands representing a part of an extended system have been important for gaining an understanding of the physical properties of artificial spin ice systems. The magnetic ordering of kagome spin ice building blocks has been extensively studied both for demagnetized states [8] and thermally relaxed ground state ordering [11] as well as thermally induced dynamics [12]. For square ice building blocks, thermal effects have been directly measured through their stray field $[13,14]$. Studies of the energy landscape and ordering of shakti lattice building blocks containing elements of two different island sizes have previously not been reported in the literature.

Several different methodologies have been applied to model artificial spin ice to gain an understanding of the energetics and underlying physics. Micromagnetics is the most accurate approach but it requires significant computational effort even for systems consisting of only a few islands. Calculations involving magnetization dynamics and incorporation of thermal effects in extended spin ice systems are computationally too demanding at this level of theory. Simpler models such as the point dipole or dumbbell representations of each island have therefore been used to calculate ground state ordering, thermal dynamics and long range interaction between islands. In the point dipole model, a magnetic dipole is placed at the center of each island and the dipole-dipole interaction 
between islands evaluated, while for the dumbbell approximation, two magnetic charges, separated by some fixed distance, are placed on each island [15-17].

The mechanism of thermally assisted transitions in such systems can be evaluated using the geodesic nudged elastic band (GNEB) method [18] where the activation energy is obtained as the energy rise along the minimum energy path for the transition. The pre-exponential in the Arrhenius rate expression can be estimated from harmonic transition state theory [19-21] as has been demonstrated for isolated islands [22] as well as elements of kagome spin ice [23]. When the islands are not too large, the magnetization can be in a single domain state and described by a single vector, the macrospin. The GNEB and harmonic transition state theory (HTST) methodology can, however, also be used to describe more general transition mechanisms where the internal structure of the island is taken into account [24].

In this article, the energetics of a shakti spin ice element consisting of eight small islands and one large island is studied. Two reversal pathways between equivalent, low energy states are studied. The point dipole model and dumbbell model with adjustable distance between the magnetic charges are compared with micromagnetic calculations for both local energy minima and configurations representing transition states.

\section{Models and methodology}

The system investigated in this study can be considered as a building block of the shakti lattice comprising one long island and eight surrounding short islands, as shown in Fig. 1. The islands are stadium shaped, $150 \mathrm{~nm}$ wide of $3 \mathrm{~nm}$ thickness and with a length of $450 \mathrm{~nm}$ (short islands) or $1050 \mathrm{~nm}$ (long islands). The lattice constant, defined as shown in Fig. 1, is taken to be $600 \mathrm{~nm}$. The islands are assumed to be composed of a soft magnetic material with magnetization of $M=200 \mathrm{kA} / \mathrm{m}$. The dimensions of the islands and materials parameters used for the modeling are similar to those previously reported in the literature.

\subsection{Micromagnetic modelling}

The energetics of the system are modeled by micromagnetic calculations performed using the software mumax3 [25]. The exchange constant is taken to be $A=13 \times 10^{-12} \mathrm{~J} / \mathrm{m}$ and the magnetization value given above. No magnetocrystalline anisotropy is included for the magnetic material composing the islands. The total magnetic moment of the islands comes out to be $4.05 \times 10^{6} \mu_{\mathrm{B}}$ for the small islands and $10.5 \times 10^{6} \mu_{\mathrm{B}}$ for the large ones.

The energy associated with the macrospin of each island includes a shape anisotropy term, $E_{a}=K \cos (\theta)^{2}$, where $\theta$ is the angle between the magnetization and the long axis, in addition to the total sum of magnetostatic interaction with all the other macrospins in the system. The micromagnetic model is used to evaluate this anisotropy term which is added to the dipole and dumbbell models. The anisotropy parameter $K$ is determined by calculating the energy increase when the macrospin of an island is rotated from the low energy state with the magnetization pointing along the long axis of the island to the elevated energy state where the magnetization is pointing perpendicular to the long axis. In both cases, the macrospin vector is in the plane of the island. This shape anisotropy term corresponds to $K=0.69 \mathrm{eV}$ and $1.78 \mathrm{eV}$ for the short and long islands, respectively.

\subsection{Point dipole model}

In the point dipole model, the energy of the system is approximated as

$$
E_{d-d}=E_{a}-M^{2} \sum_{i} \sum_{j>i} V_{i} V_{j}\left(\frac{\vec{m}_{i} \cdot \vec{m}_{j}}{\left|\vec{r}_{i j}\right|^{3}}-\frac{3 \vec{m}_{i} \cdot \vec{r}_{j i} \vec{m}_{j} \cdot \vec{r}_{i j}}{\left|\vec{r}_{i j}\right|^{5}}\right)
$$

where $V_{i}$ is the volume of island $i, M$ is the magnetization per volume, $m_{i}$ a unit vector giving the direction of the magnetic moment of island $i, \vec{r}_{i}$ is the location of the center of island $i$ and $\vec{r}_{i j}=\vec{r}_{j}-\vec{r}_{i}$.

\subsubsection{Dumbbell model. In the dumbbell model, the energy of the system is approximated as}

$$
E_{d b}=E_{a}-M^{2} \sum_{i} \sum_{j>i} \frac{V_{i} V_{j}}{d_{i} d_{j}}\left(\frac{1}{\left|\vec{r}_{p_{i}}-\vec{r}_{p_{j}}\right|}-\frac{1}{\left|\vec{r}_{p_{i}}-\vec{r}_{n_{j}}\right|}-\frac{1}{\left|\vec{r}_{n_{i}}-\vec{r}_{p_{j}}\right|}+\frac{1}{\left|\vec{r}_{n_{i}}-\vec{r}_{n_{j}}\right|}\right)
$$

where $\vec{r}_{p_{i}}$ and $\vec{r}_{n_{i}}$ are the locations of the positive and negative charges associated with island $i$, placed symmetrically along the long axis of the island, and $d_{i}$ is the distance between them. We introduce here an adjustable parameter in the dumbbell model, namely the ratio of the distance and the length of the island, $d / L$. Traditionally, $d$ has been assumed to be equal the length of the island, i.e. the magnetic charges are placed at opposite ends of the islands. As we demonstrate below, this leads to an overestimation of the interaction. The scaling parameter $d / L$ is thus introduced to adjust the strength of the interaction and match the results of the micromagnetic calculations. The same ratio is used for the small and the large islands. For a small distance between the two magnetic charges, 

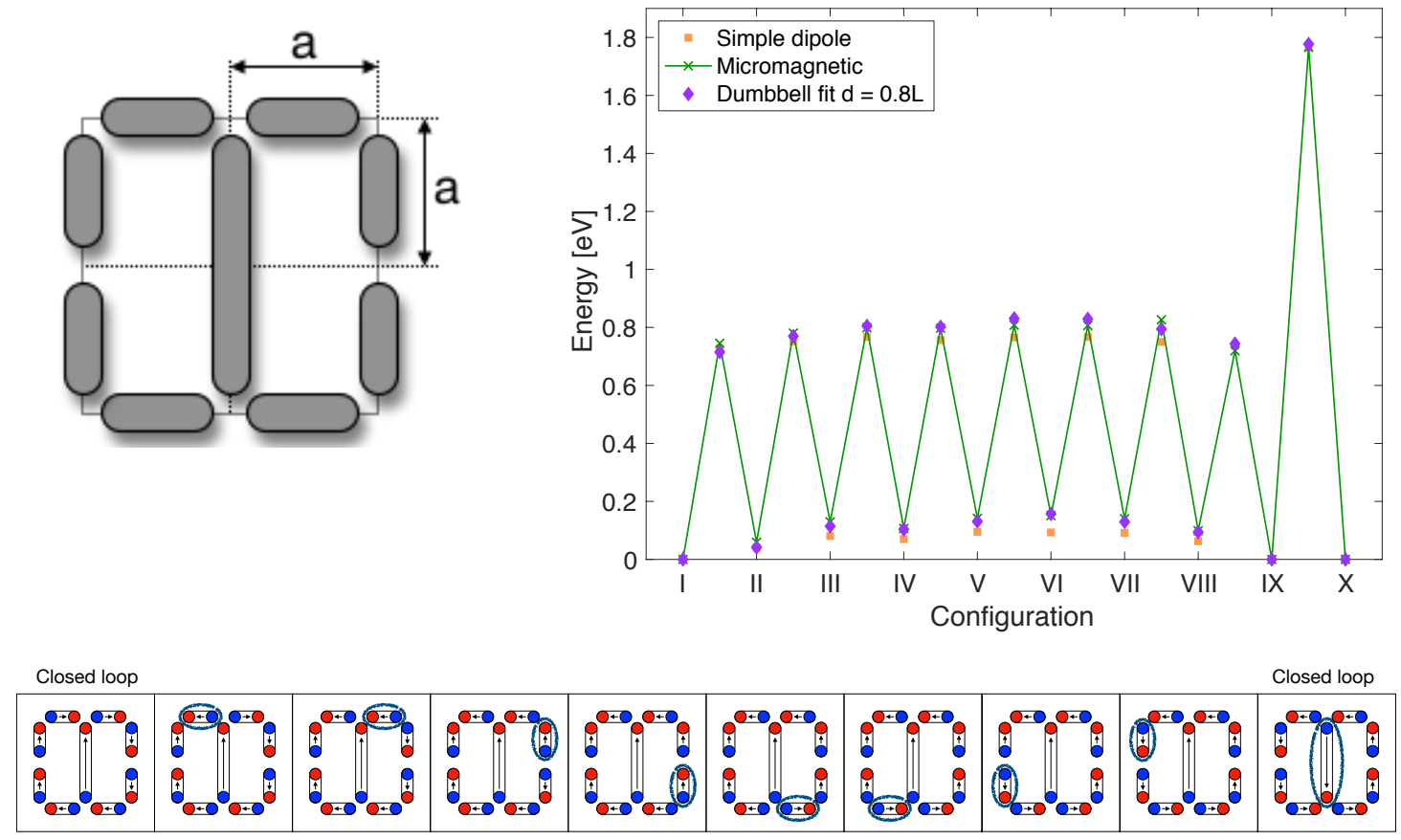

FIG. 1. Top left: An illustration of the shakti lattice building block studied here showing the definition of the lattice parameter, $a$. The long island has the same nearest neighbor distance as the shorter islands. Top right: Comparison of micromagnetic, dipole and scaled dumbbell model calculations of the energy along a transition path from one low energy, closed loop state to another equivalent one with opposite orientation of the magnetic moments. The point dipole model underestimates while the dumbbell model overestimates the interaction energy. By reducing the distance between the two magnetic charges to 0.8 of the island length, $d=0.8 \mathrm{~L}$, close agreement is obtained between the dumbbell and micromagnetic results. Bottom: Schematic illustration of the arrangement of the magnetic moments along the transition path between the two closed loop ground states. The island where the macrospin has been reversed in going from left to right is circled.

$d / L<0.1$, the dumbbell model gives results that are close to those of the point dipole model. For larger distances, the dumbbell model includes the effect of higher order multipoles in the multipole expansion of the magnetic field of an island. As a result, the dumbbell model gives stronger interaction between the islands than the point dipole model.

\section{Results}

The calculations of the shakti element are started with each macrospin pointing along the long axis of the corresponding island, forming either in a closed loop configuration, where the macrospins of all the short islands are arranged head to tail (shown in Fig. 1) or in a so-called onion configuration (shown in Fig. 2). The orientation of the macrospins is then allowed to change in order to minimize the energy of the system. This relaxation turns out to result in only a minor change in the orientation of the macrospins and the energy of the system. In the micromagnetic calculations, the closed loop state is found to be slightly lower in energy than the onion state, by $0.05 \mathrm{eV}$.

The GNEB method is used in combination with the point dipole model to find an optimal transition path between the two closed loop states as well as an optimal transition path between the two onion states. The resulting paths determined from these calculations are shown in Fig. 1,2. For each island, there are two possibilities for rotating the macrospin, either inward or outward. Depending on the arrangement of the macrospins of the neighboring island, one could have a lower energy barrier than the other. The figure shows the energy for the rotation direction that corresponds to the lower energy barrier, but in all cases the difference is small. The energy along these paths is then evaluated using also the micromagnetic and the dumbbell models. 

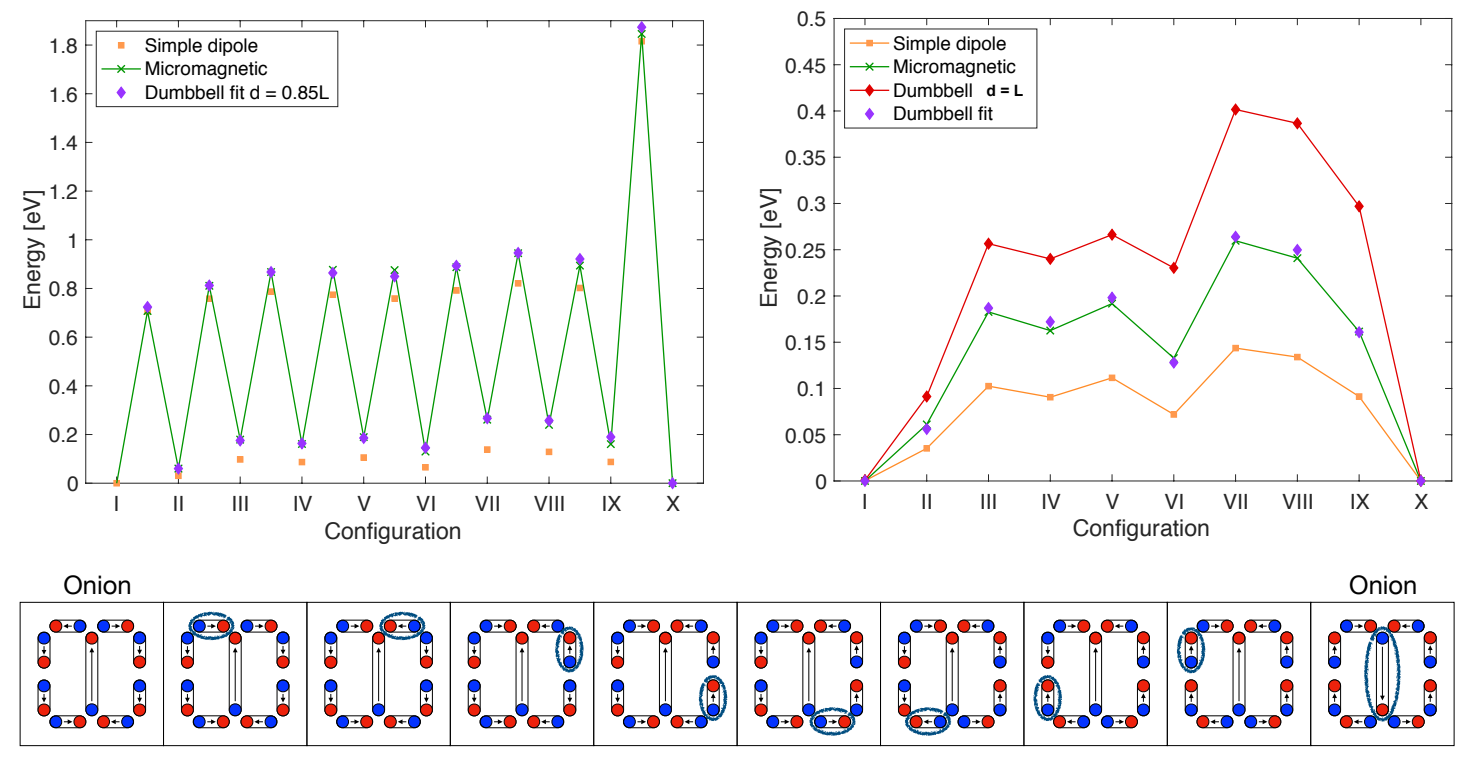

FIG. 2. Top left: Comparison of micromagnetic, dipole and scaled dumbbell model calculations of the energy along a transition path from one onion state to another equivalent one with opposite orientation of the magnetic moments. The point dipole model underestimates while the dumbbell model overestimates the interaction energy. By reducing the distance between the two magnetic charges, $d=0.85 \mathrm{~L}$, close agreement is obtained between the dumbbell and micromagnetic results. Top right: A zoom in on the energy of the local minima, same as on the graph to the left. The overestimate of the interaction energy when the magnetic charges are placed at the end of the islands, $d=L$, is about as large as the underestimation by the point dipole model. Bottom: Schematic illustration of the arrangement of the magnetic moments along the transition path between the two onion states. The island where the macrospin has been reversed in going from left to right is circled.

Figures 1, 2 show the calculated results for the transition paths between the two closed loop states (see Fig. 1) and between the two onion states (see Fig. 2). Both the energy of the various states as well as the estimated activation energy for macrospin rotations are shown. The activation energy for the rotation of the large island macrospin occurs last in these paths and is clearly much larger than the activation energy for the small islands. The point dipole model is found to underestimate the interaction energy. Both the energy of the various local energy minima and the estimates of the energy barriers are lower than the micromagnetic predictions. The dumbbell model, however, overestimates the interaction energy by a similar amount (see results for $d=L$ in the top right of Fig. 2). However, by bringing the magnetic charges closer by about $20 \%$, the dumbbell model can reproduce the micromagnetic results quite well, both the energy minima and the energy barriers.

\section{Conclusions}

The calculated results presented here show that the point dipole model can underestimate the interaction between islands in spin ice systems, while the dumbbell model gives an overestimation. A modified dumbbell model where the distance between the magnetic poles in an island is smaller than the length of the island, in this case by ca. $20 \%$, can however reproduce the results of micromagnetic calculations quite closely. Not only is the energy of the various states reproduced well, but also the energy barriers for transitions. The same parameter value was used for the large and small islands in the shakti lattice element studied here, but it remains to be seen how general the optimal value of this parameter is for the large variety of islands used in spin ice systems.

In order to simulate the long time scale evolution of spin ice systems, it is important to have a model for the energetics that does not require excessive computational effort. The modified dumbbell model with a scaled distance parameter appears to provide a good choice. The mechanism and rate of transitions can then be calculated using saddle point searches with the adaptation of the minimum mode following method to magnetic systems [26] and long time scale evolution simulated with the adaptive kinetic Monte Carlo method [27,28]. 


\section{Acknowledgments}

This work was funded by Icelandic Research Fund (grants no. 152483-051, 185405-051, 163048-053 and 184949-051), University of Iceland Research Fund and Russian Foundation of Basic Research (grant RFBR 18-0200267 A). The calculations were performed on resources provided by the Icelandic High Performance Computing Centre at the University of Iceland.

\section{References}

[1] Wang R.F., Nisoli C., Freitas R.S., Li J., McConville W., Cooley B.J., Lund M.S., Samarth N., Leighton C., Crespi V.H. and Schiffer P. Artificial 'spin ice' in a geometrically frustrated lattice of nanoscale ferromagnetic islands. Nature, 2006, 439, P. 303-306.

[2] Nisoli C., Moessner R. and Schffer P. Colloquium: Artificial spin ice: Designing and imaging magnetic frustration. Rev. Mod. Phys., 2013, 85, P. 1473-1490

[3] Kapaklis V., Arnalds U.B., Farhan A., Chopdekar R.V., Balan A., Scholl A., Heyderman L.J. and Hjörvarsson B. Thermal fluctuations in artificial spin ice. Nat. Nanotech., 2014, 9, P. 514-519.

[4] Farhan A., Scholl A., Petersen C.F., Anghinolfi L., Wuth C., Dhuey S., Chopdekar R.V., Mellado P., Alava M.J. and van Dijken S. Thermodynamics of emergent magnetic charge screening in artificial spin ice. Nat. Commun., 2016, 7, P. 12635 (6 pp).

[5] Arnalds U.B., Chico J., Stopfel H., Kapaklis V., Bärenbold O., Verschuuren M.A., Wolff U., Neu V., Bergman A. and Hjörvarsson B. A new look on the two-dimensional Ising model: thermal artificial spins. New J. Phys., 2016, 18, P. 023008 (8 pp).

[6] Chern G.-W., Morrison M.J. and Nisoli C. Degeneracy and Criticality from Emergent Frustration in Artificial Spin Ice. Phys. Rev. Lett., 2013, 111, P. 177201 (5 pp).

[7] Morrison M.J., Nelson T.R. and Nisoli C. Unhappy vertices in artificial spin ice: New degeneracies from vertex frustration. New J. Phys., 2013, 15, P. 045009 (23 pp).

[8] Mengotti E., Heyderman L.J., Rodríguez F., Bisig A., Le Guyader L., Nolting F. and Braun H.-B. Building blocks of an artificial kagome spin ice: Photoemission electron microscopy of arrays of ferromagnetic islands. Phys. Rev. B, 2008, 78, P. 144402 (7 pp).

[9] Gilbert I., Chern G.-W., Zhang S., O’Brien L., Fore B., Nisoli C. and Schiffer P. Emergent ice rule and magnetic charge screening from vertex frustration in artificial spin ice. Nat. Phys., 2014, 10, P. 670-675.

[10] Stopfel H., Östman E., Chioar I.-A., Greving D., Arnalds U.B., Hase T.P.A, Stein A., Hjörvarsson B. and Kapaklis V. Magnetic order and energy-scale hierarchy in artificial spin-ice structures. Phys. Rev. B, 2018, 98, P. 014435 (8 pp).

[11] Arnalds U.B., Farhan A., Chopdekar R.V., Kapaklis V., Balan A., Papaioannou E.T. Ahlberg M., Nolting F., Heyderman L.J. and Hjörvarsson B. Thermalized ground state of artificial kagome spin ice building blocks. Appl. Phys. Lett., 2012, 101, P. 112404 (4 pp).

[12] Farhan A., Derlet P.M., Kleibert A., Balan A., Chopdekar R.V., Wyss M., Anghinolfi L., Nolting F. and Heyderman L.J. Exploring hyper-cubic energy landscapes in thermally active finite artificial spin-ice systems. Nat. Phys., 2013, 9, P. $375-382$.

[13] Pohlit M., Porrati F., Huth M., Ohno Y., Ohno H. and Müller J. Nanocluster building blocks of artificial square spin ice: Stray-field studies of thermal dynamics. J. Appl. Phys., 2015, 117(17), P. 17C746.

[14] Pohlit M., Porrati F., Huth M., Ohno Y., Ohno H., and Müller J., Magnetic stray-field studies of a single Cobalt nanoelement as a component of the building blocks of artificial square spin ice. J. Magn. Magn. Mater, 2016, 400, P. $206-212$.

[15] Chern G.-W., Mellado P. and Tchernyshyov O., Two-Stage Ordering of Spins in Dipolar Spin Ice on the Kagome Lattice. Phys. Rev. Lett., 2011, 106, P. 207202 (4 pp).

[16] Castelnovo C., Moessner R., and Sondhi S.L. Magnetic monopoles in spin ice. Nature, 2008, 451, P. 42-45.

[17] Möller G. and Moessner R. Magnetic multipole analysis of kagome and artificial spin-ice dipolar arrays. Phys. Rev. B, 2009, 80, P. 140409 (4 pp).

[18] Bessarab P.F., Uzdin V.M. and Jónsson H. Method for finding mechanism and activation energy of magnetic transitions, applied to skyrmion and antivortex annihilation. Comp. Phys. Commun., 2015, 196, P. 335-347.

[19] Bessarab P.F., Uzdin V.M. and Jónsson H. Harmonic transition state theory of thermal spin transitions. Phys. Rev. B, 2012, 85, P. 184409 (4 pp).

[20] Bessarab P.F., Uzdin V.M. and Jónsson H. Potential energy surfaces and rates of spin transitions. Z. Phys. Chem., 2013, 227, P. $1543-1557$.

[21] Liashko S.Y., Uzdin V.M., Jónsson H. Thermal stability of magnetic states in submicron magnetic islands. Nanosystems: Physics, Chemistry, Mathematics, 2017, 8(5), P. 572-578.

[22] Liashko S.Y., Jónsson H., Uzdin V.M. Calculations of switching field and energy barrier for magnetic islands with perpendicular anisotropy. Nanosystems: Physics, Chemistry, Mathematics, 2017, 8(6), P. 701-708.

[23] Liashko S.Y., Jónsson H., Uzdin V.M. The effect of temperature and external field on transitions in elements of kagome spin ice. New J. Phys., 2017, 19, P. 113008 (8 pp).

[24] Bessarab P.F., Uzdin V.M. Jónsson H. Size and shape dependence of thermal spin transitions in nanoislands. Phys. Rev. Lett., 2013, 110(2), P. 020604 (5 pp).

[25] Vansteenkiste A., Leliaert J., Dvornik M., Helsen M., Garcia-Sanchez F. and Van Waeyenberge B. The design and verification of MuMax3. AIP Advances, 2014, 4, P. 107133 (22 pp).

[26] Müller G., Bessarab P.F., Vlasov S.M., Lux F., Kiselev N.S., Blügel S., Uzdin V.M. and Jónsson H. Duplication, collapse and escape of magnetic skyrmions revealed using a systematic saddle point search method. Phys. Rev. Lett., 2018, 121, P. 197202 (5 pp).

[27] Henkelman G. and Jónsson H. Long time scale kinetic Monte Carlo simulations without lattice approximation and predefined event table. J. Chem. Phys., 2001, 115, P. 9657-9666.

[28] Chill S.T., Welborn M., Terrell R., Zhang L., Berthet J.-C., Pedersen A., Jónsson H., and Henkelman G. EON: Software for long time simulations of atomic scale systems. Modelling Simul. Mater. Sci. Eng., 2014, 22, P. 055002 (16 pp). 